\title{
Automated pollen identification using microscopic imaging and texture analysis
}

\author{
J. Victor $\operatorname{Marcos}^{\mathrm{a}, *}$, Rodrigo Nava ${ }^{\mathrm{b}}$, Gabriel Cristóbal ${ }^{\mathrm{a}}$, Rafael Redondo ${ }^{\mathrm{a}, \mathrm{c}}$, \\ Boris Escalante-Ramírez ${ }^{\mathrm{b}}$, Gloria Bueno ${ }^{\mathrm{c}}$, Óscar Déniz ${ }^{\mathrm{c}}$, Amelia \\ González-Porto $^{\mathrm{d}}$, Cristina Pardo ${ }^{\mathrm{e}}$, Francois Chung ${ }^{\mathrm{f}}$, Tomás Rodríguez ${ }^{\mathrm{f}}$ \\ ${ }^{a}$ Instituto de Óptica, Spanish National Research Council (CSIC), Serrano 121, Madrid, \\ Spain \\ ${ }^{b}$ Posgrado en Ciencia e Ingeniería de la Computación, Universidad Nacional Autónoma \\ de México, Mexico City, Mexico \\ ${ }^{c}$ VISILAB Department at the University of Castilla La Mancha, Av. Camilo José Cela \\ $s / n$, Ciudad Real, Spain \\ ${ }^{d}$ Centro Agrícola Marchamalo, Guadalajara, Spain \\ ${ }^{e}$ Facultad de Farmacia, Univ. Complutense, Madrid, Spain \\ ${ }^{f}$ Inspiralia, Estrada 10, Madrid, Spain
}

\begin{abstract}
Pollen identification is required in different scenarios such as prevention of allergic reactions, cilmate analysis or apiculture. However, it is a timeconsuming task since experts are required to recognize each pollen grain through the microscope. In this study, we performed an exhaustive assesment on the utility of texture analysis for automated characterization of pollen samples. A database composed of 1800 brightfield microscopy images of pollen grains from 15 different taxa was used for this purpose. A pattern

\footnotetext{
*Corresponding author. Phone: 0034915616800

Email addresses: jvmarcos@gmail.com (J. Victor Marcos), urielrnv@uxmcc2.iimas.unam.mx (Rodrigo Nava), gabriel@optica.csic.es (Gabriel Cristóbal), rafa@optica.csic.es (Rafael Redondo), boris@servidor.unam.mx (Boris Escalante-Ramírez), Gloria.Bueno@uclm.es (Gloria Bueno), Oscar.Deniz@uclm.es (Óscar Déniz), avgonzalez@externas.jccm.es (Amelia González-Porto), cpardo@farm.ucm.es (Cristina Pardo), francois.chung@inspiralia.com (Francois Chung), tomas.rodriguez@inspiralia.com (Tomás Rodríguez)
} 
recognition-based methodology was adopted to perform pollen classification. Four different methods were evaluated for texture feature extraction from the pollen image: Haralick's Gray-Level Co-occurrence Matrices (GLCM), LogGabor Filters (LGF), Local Binary Patterns (LBP) and Discrete Tchebichef Moments (DTM). Fisher's discriminant analysis and $k$-nearest neighbour were subsequently applied to perform dimensionality reduction and multivariate classification, respectively. Our results reveal that LGF and DTM, which are based on the spectral properties of the image, outperformed GLCM and LBP in the proposed classification problem. Furthermore, we found that the combination of all the texture features resulted in the highest performance, yielding an accuracy of $94.83 \%$. Therefore, thorough texture characterization could be considered in further implementations of automatic pollen recognition systems based on image processing techniques.

Keywords: Texture analysis, Pollen identification, Gray-Level

Co-occurrence Matrix, Log-Gabor Filters, Local Binary Patterns, Discrete Tchebichef Moments 


\section{Introduction}

Palynology is the study of pollen grains produced by seed plants and spores (Erdtman et al., 1969). Accurate identification of pollen types is a relevant issue in several scenarios. For instance, quantifying the concentration of airborne pollen may help people suffering from allergic reactions to adopt adequate treatment strategies. In archaeology, pollen fossils are analyzed to reconstruct ecological and climate conditions during past periods. For commercial purposes, pollen is involved in the search of oil and gas. In apiculture, pollen classification is required to identify nectar sources, which determine the quality of the product and enable the authentication of its origin (Kaya et al., 2013). Currently, pollen identification is based on visual inspection of microscopy images. It is a time-consuming and costly procedure since a trained expert must manually classify each pollen grain (Mitsumoto et al., 2009). Furthermore, a subjective result is obtained as it depends on the expert's criterion. Thus, automated methods for pollen identification are required in order to overcome the limitations of the conventional procedure (Stillman and Flenley, 1996).

Slides observed through the microscope contain a variable number of pollen grains. Therefore, automatic pollen identification would involve segmentation and classification tasks (Kumar et al., 2010). Segmentation aims to localize each of the pollen grains in the slide, separating it from the rest of the content. In classification, the isolated pollen grain is assigned to one of a predefined set of categories (taxa). The present study is focused on the latter. For this purpose, brightfield microscopy images corresponding to a subset of honey-bee pollen taxa commonly found in the mediterranean area 
were analyzed. Previous studies in the field of automated palynology focused on this specific type of pollen samples (Chica, 2012; Carrión et al., 2004).

A pattern recognition approach was adopted to model the pollen grain classification problem. Hence, it is assumed that grains from a given taxon own distinctive characteristics with respect to samples from other taxa (Duda et al., 2012). Pattern recognition mainly involves feature extraction and multivariate pattern classification (Bishop, 1995). The former consists in the definition of a set of quantitative measurements, called features or descriptors, capturing representative attributes of the image (pollen grain) to be identified. As a result, the grain is described by a point in the multivariate space defined by the feature set. Subsequently, a multivariate classifier is then used to define decision boundaries between categories in the input feature space.

Diverse features have been previously evaluated in automated classification of pollen taxa based on image processing techniques. Morphological and geometric descriptors have been the most common choice for this purpose (Kaya et al., 2013; Mitsumoto et al., 2009; Boucher et al., 2002; Treloar et al., 2004; Chica, 2012). They include measurements such as area, perimeter, concavity, convexity or circularity of the grain. Also, the utility of pore detection has been evaluated (Chen et al., 2006). However, it was reported that similar pore appearances can be found in samples from different taxa, reducing the discriminative capability of this approach (Chen et al., 2006). Additionally, texture attributes of the grain's surface or exine have been suggested as pollen descriptors. Pollen recognition from texture analysis is based on the differences found between the ornamentation of the exines from 
distinct taxa. Previously, texture descriptors provided promising results in pollen identification both separately (Kumar et al., 2010; Langford et al., 1990; Li and Flenley, 1999; Carrión et al., 2004; Li et al., 2004; Zhang et al., 2005) and combined with morphological features (Chen et al., 2006; Zhang et al., 2004; Rodriguez-Damian et al., 2006; Punyasena et al., 2012).

In particular, texture represents an efficient approach for pollen identification as it provides several advantages. As the rest of the techniques based on image processing, texture methods could be integrated in a software capable of automatically assessing large amounts of pollen samples. In addition, texture analysis enables the inspection of broken grains typically found in fossil pollen samples for which morphological descriptors would be inappropriate. Furthermore, in contrast to methods based on pore detection, texture properties do not depend on the position of the grain in the slide. Despite the benefits of the texture-based approach, a reduced number of texture methods have been applied to pollen characterization. Commonly, previous studies focused on pollen texture used conventional first-order and second-order statistical features, with the latter being computed from Haralick's co-occurrence analysis (Langford et al., 1990; Li et al., 2004; Chen et al., 2006; Rodriguez-Damian et al., 2006). Occasionally, other techniques included Laws masks (Li and Flenley, 1999; Carrión et al., 2004) and Gabor filters (Zhang et al., 2004). On the other hand, a small number of taxa, typically lower than 10, was analyzed in these preceding research works. Therefore, further analysis including other different methods and a higher variety of taxa is required to obtain solid conclusions about the potential of texture in pollen identification. 
The purpose of the present study is to perform an exhaustive analysis on the correspondence between the texture of a pollen grain and its taxon. Texture is a property related to the roughness of a surface and is characterized by the variation of pixel intensity in the spatial domain (Tuceryan and Jain, 1993). A number of texture descriptors have been proposed in the literature. Tuceryan and Jain (Tuceryan and Jain, 1993) grouped them into four types: statistical, geometrical, model-based and signal processing. In our study, we selected a subset of texture methods in order to capture different properties of the pollen texture. Haralick's Gray-Level Co-occurrence Matrices (GLCM) (Haralick et al., 1973) were used as a reference method in our experiments since it has been adopted by other researchers (Langford et al., 1990; Li et al., 2004; Chen et al., 2006; Rodriguez-Damian et al., 2006). In addition, we evaluated the utility of other techniques that have not been previously applied to texture-based pollen classification namely Log-Gabor Filters (LGF) (Field et al., 1987), Local Binary Patterns (LBP) (Ojala et al., 2002) and Discrete Tchebichef Moments (DTM) (Mukundan et al., 2001). These methods have provided satisfactory results in other texture and pattern recognition problems (Soh and Tsatsoulis, 1999; Gao et al., 2007; Das et al., 2013; Marcos and Cristóbal, 2013).

This paper is organized as follows. The second section describes the acquisition of pollen images as well as the database employed in our research. In the third section, the applied methodology is presented. An explanation of the techniques used for image segmentation and preprocessing, texture feature extraction, dimensionality reduction and pattern classification is provided. The fourth section of the paper includes the results achieved in our 
experiments as well as an analysis of them. Finally, in the last section, the main findings of the study are highlighted and discussed.

\section{Materials}

\subsection{Pollen dataset}

Slide images were captured using a NIKON E200 microscope and a camera NIKON DS-Fi1. Images of isolated pollen grains were obtained manually. The complete procedure for image acquisition comprised several steps. Balls of pollen collected by bees were dissolved with glycerogelatin drops and prepared in slides sealed with a coverslip. A 40x magnification was used to acquire images of the slide containing several pollen grains. The acquisition consisted in stacks with 31 images of the slide in order to ensure an optimum focus. The best focused slide was identified by an expert. Subsequently, pollen grains were manually extracted from it by defining a rectangular region. As a result, the dimensions of the pollen images varied from one to another. Pollen samples from 15 different taxa from the mediaterranean area were captured: 1) Aster, 2) Brassica, 3) Campanulaceae, 4) Carduus, 5) Castanea, 6) Cistus, 7) Cytisus, 8) Echium, 9) Ericaceae, 10) Helianthus, 11) Olea, 12) Prunus, 13) Quercus, 14) Salix and 15) Teucrium. The database analyzed in this study was composed of 120 brightfield microscopy images per pollen taxon, resulting in a total of 1800 images. Figure 1 depicts an example for each taxon included in our research. 

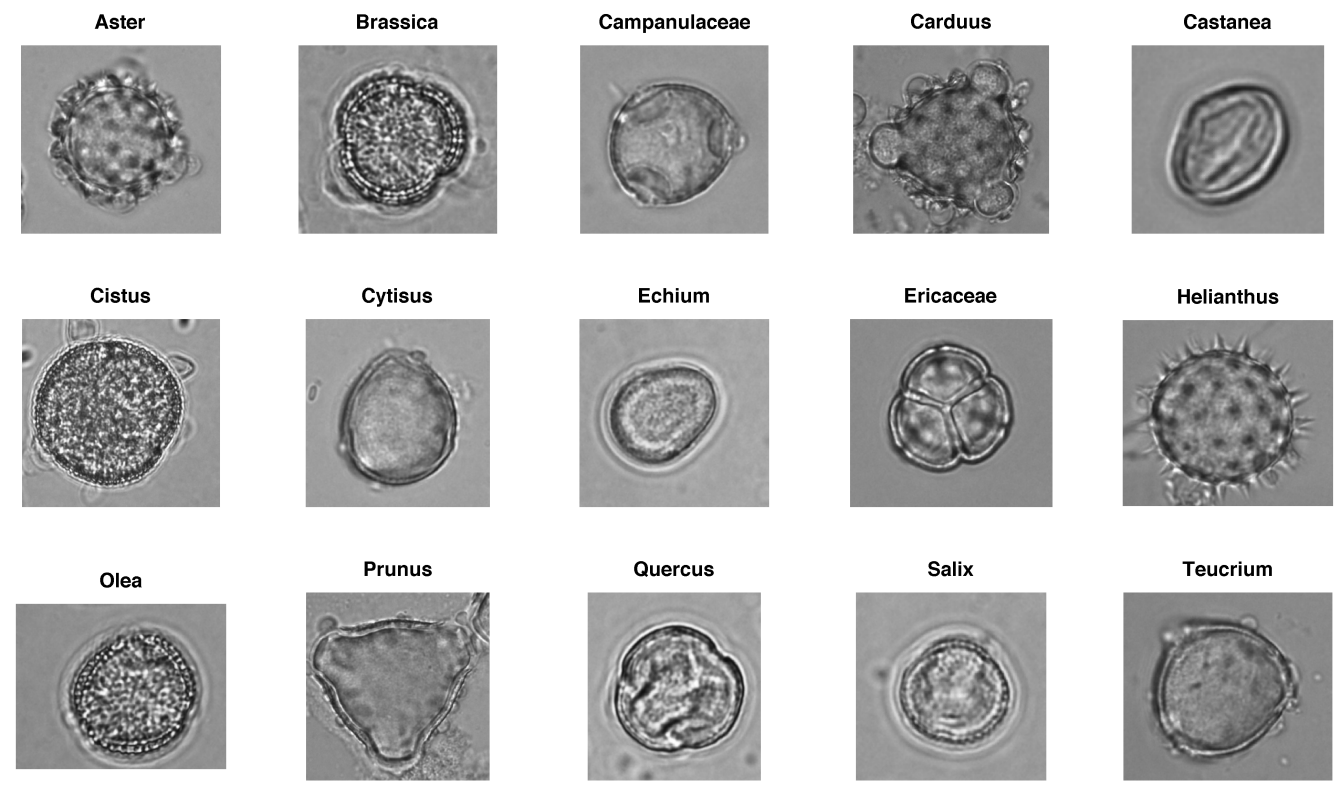

Figure 1: Sample images of the pollen taxa analyzed in our study.

\section{Methods}

In our study, the discriminant capability of texture in automatic pollen identification is evaluated. Hence, pollen grain images are assigned to one of several categories (taxa) according to their texture properties. We propose a pattern recognition-based methodology to perform such a classification task. It is composed of four different stages: segmentation and preprocessing, texture feature extraction, dimensionality reduction and classification. Figure 2 provides an schematic diagram of the proposed methodology.

\subsection{Segmentation and preprocessing}

The texture of a pollen grain is given by the elements characterizing its surface or exine. In order to perform texture analysis of the pollen image, segmentation was carried out to separate the target object (i.e., the grain) 


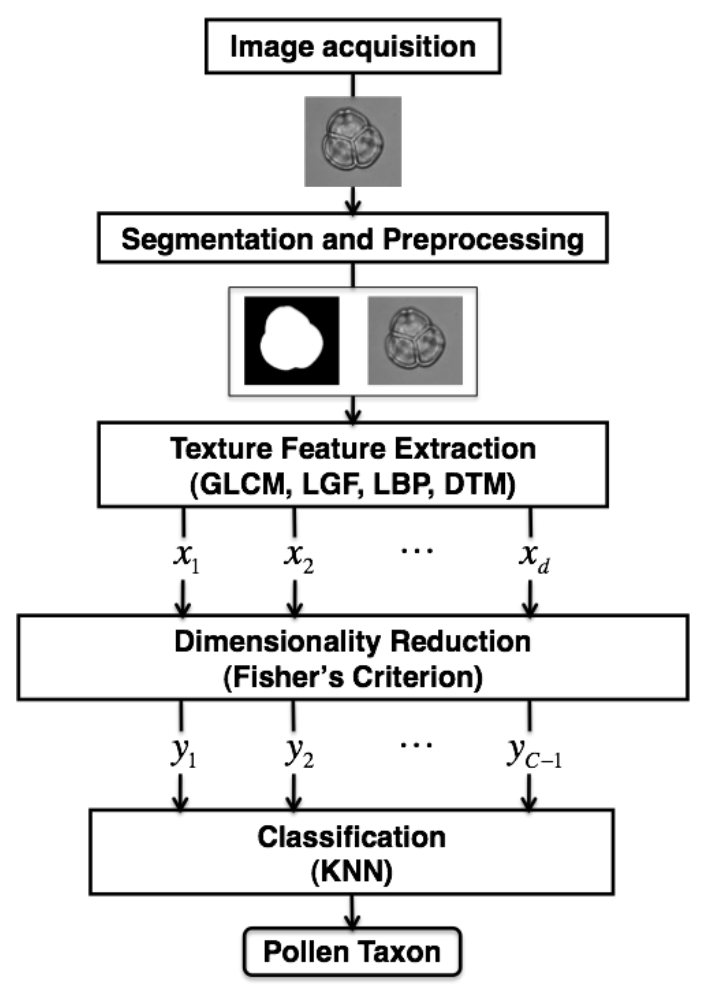

Figure 2: A scheme of the methodology adopted for automatic identification of the pollen taxon based on texture analysis. From the input image, four main processing stages are identified: segmentation and preprocessing, texture feature extraction, dimensionality reduction and classification. 


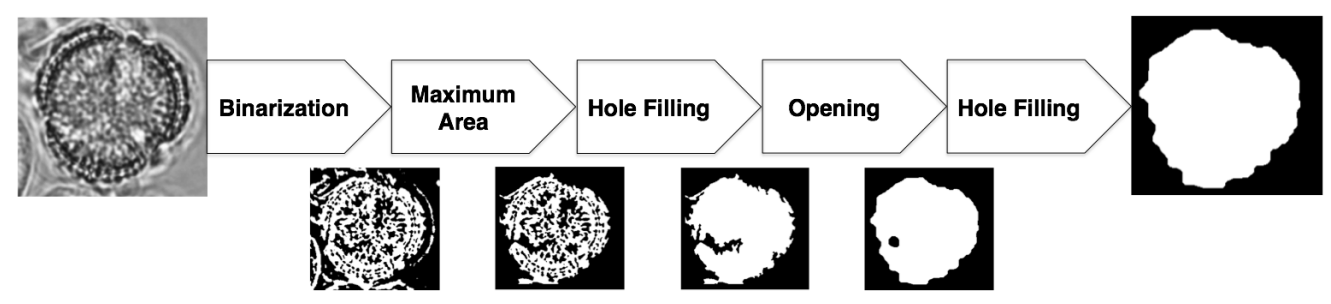

Figure 3: Segmentation proccess for the identification of the pollen grain in the input image.

from the background. A binary mask was obtained for each pollen image, indicating those pixels contained in the texture of interest. Segmentation involved several steps:

1. Binarization. The image was thresholded using an Otsu-based method (Otsu, 1975).

2. Maximum area. The region with the biggest area was retained.

3. Hole filling. Any hole in the selected region was filled.

4. Opening. The border of the region was defined using erosion and dilation operators.

5. Hole filling. Any remaining hole was filled.

Figure 3 illustrates each step of the segmentation process for one of the images in our dataset.

In addition to binary segmentation, preprocessing was applied before texture feature extraction from the pollen image. Preprocessing is intended as a normalization stage that produces a new version of the image, so that it can be effectively processed by any of the four considered texture methods (GLCM, LBP, LGF and DTM). To this end, different requirements had to 
be addressed in the preprocessing stage due to the distinct nature of these methods. First, unlike GLCM and LBP, which involve the evaluation of each image pixel individually, LGF and DTM perform global analysis of the image by simultaneously processing the entire set of pixels (Field et al., 1987; Mukundan et al., 2001). Thus, for a fair comparison (classification) of the extracted features, all the images in the database must have the same dimension. Second, square images are recommended for an effective evaluation using LGF and DTM. Indeed, this assumption was made for the definition of the DTM-based texture descriptor employed in our study (Marcos and Cristóbal, 2013). Moreover, square images enable the use of the same number of scales for each direction in LGF banks. Third, LGF analysis is performed in the frequency domain from the spectral representation of both the image and the corresponding bank of filters. Hence, power-of-two sized images are required to optimize the performance of the method. Fourth, the size of the images must be properly limited as it influences the computational load of the evaluated texture methods. This is specially relevant for those methods based on a pixel-by-pixel analysis of the image like GLCM and LBP.

To fulfill these requirements, the first operation of the preprocessing stage consisted in trimming each image in the dataset, and its corresponding mask, to remove the maximum quantity of background. Subsequently, the largest image dimension $\left(L_{0}\right)$ (either rows or columns) among the trimmed images was identified. It determines the minimum size of a square capable of including any of the pollen grains in the dataset. Once $\left(L_{0}\right)$ has been obtained, the following steps were successively applied to each of the original pollen images: 
1. Trimming. Both the image and its binary mask were trimmed.

2. Background removal. The trimmed image was multiplied by its corresponding mask to set background pixels to 0 .

3. Mean removal. The mean value of the pixels in the region of interest was substracted, resulting in a zero-mean texture.

4. Image padding. New background pixels (rows and columns) with zero value were added in order to obtain an image of size $L_{0} \times L_{0}$.

5. Resizing. The image was resized to $L \times L$ pixels, where $L$ is a powerof-two integer.

We found $L_{0}=450$ pixels while the final dimension $L$ was set to 128 pixels to achieve an affordable computation time. The same downsampling factor was applied to every image in the dataset. As a result, the preprocessing stage preserved the aspect ratio between the sizes of different original pollen grains. Finally, it is worth noting that the normalization implemented by preprocessing enables each of the four texture methods to process the same version of the pollen image, ensuring an unbiased comparative analysis between them.

\subsection{Feature extraction}

The feature extraction stage aims to characterize the pollen image using a set of measurements or features. This set is commonly referred to as a feature pattern (Duda et al., 2012). In our study, texture features were employed to define representative attributes of the pollen taxa in order to perform classification. The utility of the texture attributes captured by means of four different methods was evaluated: GLCM, LGF, LBP and DTM. 


\subsubsection{Gray-level co-occurrence matrices (GLCM)}

GLCM analysis is based on the assumption that texture information is contained in the spatial relationship between gray levels (Haralick et al., 1973). For the computation of GLCM, suppose that each pixel $I(x, y)$ in the image is assigned to one of $B$ gray levels. Hence, the co-occurrence matrix $P_{i j}$ is obtained by assessing all the possible combinations between two intensity levels $(i, j=1, \ldots, B)$. Here, $P_{i j}$ represents the number of occurrences of two pixels with gray levels $i$ and $j$ separated by a distance $\delta$ in the direction determined by the angle $\phi$. Note that $P_{i j}=P_{j i}$, i.e., the occurrence of $(j, i)$ is considered as a match when computing $P_{i j}$ and vice versa. The resulting matrix is a function of both the distance and the angular direction between pixels, so that different matrices can be obtained by varying these parameters. Usually, the elements of the co-occurrence matrix are normalized in order to represent probabilities, providing the relative frequency of occurrence for a pair of gray levels. The element $p(i, j)$ denotes the probability of finding the pair of levels $(i, j)$ in the image, which is obtained as (Haralick et al., 1973):

$$
p(i, j)=\frac{P_{i j}}{\sum_{i, j}^{B} P_{i j}}
$$

These matrices of probabilities can be used to obtain statistical features that characterize the texture (Haralick et al., 1973). As suggested in (Soh and Tsatsoulis, 1999), we chose a subset of 10 features to capture texture properties. These include energy, contrast, correlation, homogeneity, entropy, autocorrelation, dissimilarity, cluster shade, cluster prominence, and maximum probability.

In our experiments, the quantization level $B$ was set to 8 as preceding 
studies focused on texture analysis using GLCM (Randen and Husoy, 1999).

For the displacement vector, four different values of the angle $\phi$ were assessed $\left(0^{\circ}, 45^{\circ}, 90^{\circ}\right.$ and $\left.135^{\circ}\right)$ (Soh and Tsatsoulis, 1999), while the distance parameter $\delta$ was set to 1, 2 or 3 . Each of these values represented a distinct scale for texture analysis. Thus, for a given value of $\delta$, a pattern $\mathrm{GLCM}_{\delta}$ composed of 40 descriptors (10 statistical features for each of the four orientations) was obtained to characterize the texture of a pollen grain. A multiscale feature pattern of dimension $120\left(\mathrm{GLCM}_{\mathrm{msc}}\right)$ was defined by combining the descriptors derived from the different values of $\delta$.

\subsubsection{Log-Gabor Filters (LGF)}

LGF were proposed by Field et al. (1987) to overcome the limitations of conventional Gabor filters. These are characterized by a maximum bandwidth restricted to approximately one octave and a non-zero DC component (Gao et al., 2007). In addition, it has been proved that LGF are more consistent with measurements of mammalian visual systems indicating that the cell responses are symmetric on the log frequency scale (Field et al., 1987; Kovesi, 1999). LGF are defined in the frequency domain as Gaussian functions shifted from the origin due to the singularity of the $\log (\cdot)$ function. They have a null DC component and can be optimized to produce filters with minimal spatial extent in an octave scale multiresolution scheme. Mathematically, LGF can be divided into two components referred to as radial and angular filters (Gao et al., 2007):

$$
\hat{G}(\rho, \theta)=\hat{G}_{\rho} \hat{G}_{\theta}=e^{-\frac{1}{2}\left[\frac{\log \left(\frac{\rho}{u_{0}}\right)}{\log \left(\frac{\alpha_{\rho}}{u_{0}}\right)}\right]^{2}} e^{-\frac{1}{2}\left[\frac{\left(\theta-\theta_{0}\right)}{\alpha_{\theta}}\right]^{2}}
$$


where $\rho$ and $\theta$ represent the polar coordinates, $u_{0}$ is the central frequency, $\theta_{0}$ is the orientation angle, and the parameters $\alpha_{\rho}$ and $\alpha_{\theta}$ determine the scale and the angular bandwidth, respectively. In our experiments, we set $\alpha_{\rho}=0.75$ and $\alpha_{\text {theta }}=\frac{\pi}{6}$ since previous studies showed that these values result in minimal overlap among scales one octave apart (Nava et al., 2012). These values resulted in a bank of 24 filters distributed in 4 scales and 6 orientations. In order to better cover the Fourier plane, even scales were rotated by a constant factor consisting of the half a distance between filter centers (Gross and Koch, 1995).

To characterize the response of the image to the LGF bank, the first four standard moments of the filtered image at each scale and orientation were computed (Schwartz et al., 2012). These moments include mean, variance, skewness and kurtosis. They quantify the central tendency, the degree of dispersion, the asymmetry and the peakedness of the distribution of samples in the filtered image. As a result, a feature vector composed of 96 elements (4 features for each of the 24 filters) was used to describe the texture of the pollen grain.

\subsubsection{Local binary patterns (LBP)}

The LBP operator assumes that texture is composed of different patterns or local spatial structures that are repeated in the image (Ojala et al., 2002). The LBP detects them by acting as a template mask that is evaluated at each image pixel. An estimate of the probability of occurrence of these structures is obtained by means of a histogram, which is used to describe the texture (Ojala et al., 2002). 
To compute the LBP of an image, a neighbourhood of $P$ pixels equally distributed on a circumference of radius $R$ around a central pixel $\left(g_{c}\right)$ is considered. The value of $g_{c}$ is taken as a threshold to obtain the LBP, which is expressed as:

$$
L B P_{P, R}\left(g_{c}\right)=\sum_{p=0}^{P-1} H\left(g_{p}-g_{c}\right) 2^{p}
$$

where $g_{p}(p=0, \ldots, P-1)$ are the values of the neighbours and $H(\cdot)$ is the Heaviside function. The formulation of $L B P_{P, R}$ yields a total of $2^{P}$ different patterns. The probability associated with each of them is estimated by means of its relative frequency, leading to a histogram with $2^{P}$ bins. However, for $P$ high enough, a large number of bins is obtained, resulting in sparse histograms.

It was observed that certain patterns, called as uniform patterns, present improved capabilities for texture discrimination (Ojala et al., 2002). Uniform patterns are characterized for a maximum of two bit transitions in their binary representation as measured by the operator $U\left(L B P_{P, R}\right)$, which is given by:

$$
\begin{array}{r}
U\left(L B P_{P, R}\left(g_{c}\right)\right)=\left|H\left(g_{P-1}-g_{c}\right)-H\left(g_{0}-g_{c}\right)\right| \\
+\sum_{p=1}^{P-1}\left|H\left(g_{p}-g_{c}\right)-H\left(g_{p-1}-g_{c}\right)\right|
\end{array}
$$

The uniform LBP $\left(L B P_{P, R}^{u n i}\right)$ is then defined as (Ojala et al., 2002):

$$
L B P_{P, R}^{u n i}\left(g_{c}\right)=\left\{\begin{array}{lc}
\sum_{p=0}^{P-1} H\left(g_{p}-g_{c}\right) & \text { if } U\left(L B P_{P, R}\left(g_{c}\right)\right) \leq 2 \\
P+1 & \text { otherwise }
\end{array}\right.
$$

As a result, $L B P_{P, R}^{u n i}$ produces a $(P+2)$-bin histogram that characterizes the pollen texture. In our study, three different configurations, $P=8-R=$ 
1, $P=16-R=2$ and $P=24-R=3$, were adopted to evaluate texture in several scales (Ojala et al., 2002). A multiscale texture descriptor ( $\mathrm{LBP}_{\mathrm{msc}}$ ) was obtained by combining the feature patterns from these three different scales.

\subsubsection{Discrete Tchebichef moments (DTM)}

The set of DTM provides a unique representation of an image $I(x, y)$ in the space spanned by Tchebichef kernels. The moment $T_{p q}(p, q=0,1, \ldots, L-1)$ of order $s=p+q$ is defined as (Mukundan et al., 2001):

$$
T_{p q}=\frac{1}{\tilde{\eta}(p) \tilde{\eta}(q)} \sum_{x=0}^{L-1} \sum_{y=0}^{L-1} \tilde{t}_{p}(x) \tilde{t}_{q}(y) I(x, y),
$$

where $\tilde{t}_{n}(x)$ is the scaled Tchebichef polynomial of degree $n$ and $\tilde{\eta}(n)$ is its squared norm (Mukundan, 2004). The function $r_{p q}(x, y)=\tilde{t}_{p}(x) \tilde{t}_{q}(y)$ denotes the two-dimensional Tchebichef kernel.

Recently, a texture descriptor has been proposed based on the properties of discrete Tchebichef kernels (Marcos and Cristóbal, 2013). From equation 6 , the magnitude of $T_{p q}$ quantifies the correlation between the original image $I(x, y)$ and the kernel $r_{p q}(x, y)$, which has an oscillating profile. Thus, this magnitude will be higher for images characterized by repetitive patterns occurring at a similar rate to $r_{p q}(x, y)$. This is a relevant property for texture analysis since texture involves the spatial repetition of intensity patterns (Tuceryan and Jain, 1993). As kernels of the same order are characterized by similar frequency content, i.e., similar varying patterns along different directions (Teh and Chin, 1988), a description of the texture attributes is obtained by assessing the dependence of the total moment magnitude on the 
kernel order $s$ (Marcos and Cristóbal, 2013):

$$
M(s)=\sum_{p+q=s}\left|T_{p q}\right|,(s=0,1, \ldots, 2 L-2) .
$$

The value of $M(s)$ evaluates the similarity between the original image and the varying patterns implemented by s-order Tchebichef kernels. The analysis based on DTM yields a vector $M(s)$ of length $2 L-1$ that describes the texture of the pollen grain.

\subsection{Dimensionality reduction}

Feature extraction enables to summarize the information in the pollen texture by means of a vector (pattern) of features. This vector could be directly used for pollen classification. However, dimensionality reduction was performed through a twofold purpose. First, it represents a normalization stage for the obtained feature vector since its dimension depends on the method adopted for texture analysis. Dimensionality reduction avoids this dependence by providing a new vector whose dimension can be controlled by the user. As the new dimension is smaller than that of the original feature vector, a more compact representation of the pollen texture is obtained. Second, reducing the dimension of the input feature space prevents overfitting. As detailed in (Bishop, 1995), in order to obtain an accurate statistical description of the problem, the size of the training set should be exponentially increased with the dimensionality of the input space. Commonly, a limited dataset is available in real applications such as the proposed pollen classification problem. Thus, reduced dimensionality may be advantageous for the posterior classification stage. 
We used the conventional Fisher's discriminant analysis (FDA) to perform dimensionality reduction (Fisher, 1936; Bishop, 1995). For a multiclass classification problem with $C$ possible categories, the original feature vector $\mathbf{x}=\left(x_{1}, x_{2}, \ldots, x_{d}\right)$ of dimension $d(d \geq C)$ is mapped onto a new space of dimension $d^{\prime}=C-1$. The transformation matrix $\mathbf{W}$ is obtained according to the Fisher's criterion, which maximizes the ratio of the interclass variability to the intraclass variability for the transformed samples. Mathematically, it is expressed as follows (Bishop, 1995):

$$
J(\mathbf{W})=\frac{\left|\mathbf{W}^{T} \mathbf{S}_{B} \mathbf{W}\right|}{\left|\mathbf{W}^{T} \mathbf{S}_{W} \mathbf{W}\right|}
$$

where $\mathbf{S}_{B}$ and $\mathbf{S}_{W}$ are the between-class and the within-class scatter matrices, respectively. The columns of $\mathbf{W}$ are given by the eigenvectors associated with the $d^{\prime}$ largest eigenvalues of the matrix $\mathbf{S}_{W}^{-1} \mathbf{S}_{B}$. Therefore, the projection $\mathbf{y}$ of the original feature vector $\mathbf{x}$ is obtained as (Bishop, 1995):

$$
\mathbf{y}=\mathbf{W}^{T} \mathbf{x} .
$$

\subsection{Classification}

In the final stage, the vector $\mathbf{y}$ resulting from dimensionality reduction is labelled as one of the 15 pollen classes considered in our study. According to the Bayes' decision rule, it must be assigned to the class $\omega_{i}$ for which the posterior probability $p\left(\omega_{i} \mid \mathbf{y}\right)(i=1, \ldots, C)$ is the highest in order to minimize the risk of misclassification. Multivariate analysis was performed to define decision boundaries in the $d^{\prime}$-dimensional $\left(d^{\prime}=C-1\right)$ input space using the $k$-nearest neighbour (KNN) approach. KNN is based on the approximation 
of the probability density function of a variable from a finite set of samples as described in the following expression (Bishop, 1995):

$$
p(\mathbf{y}) \approx \frac{K}{N V}
$$

where $K$ represents the total number of samples that are found in a volume $V$ centred on $\mathbf{y}$. In a classification problem, this procedure can be used to model the class-conditional density $p\left(\mathbf{y} \mid \omega_{i}\right)$ of each category $\omega_{i}$ as:

$$
p\left(\mathbf{y} \mid \omega_{i}\right) \approx \frac{K_{i}}{N_{i} V}
$$

where $K_{i}$ represents the total number of samples belonging to class $\omega_{i}$ that are found in a volume $V$ centred on $\mathbf{y}$, given that $N_{i}$ is the total number of training samples of class $\omega_{i}$.

Since the prior probability of a class is estimated as the proportion of samples belonging to that class, i.e., $p\left(\omega_{i}\right) \approx \frac{N_{i}}{N}$, the posterior probability $p\left(\omega_{i} \mid \mathbf{y}\right)$ can be obtained from the Bayes' theorem as follows (Bishop, 1995):

$$
p\left(\omega_{i} \mid \mathbf{y}\right)=\frac{p\left(\mathbf{y} \mid \omega_{i}\right) p\left(\omega_{i}\right)}{p(\mathbf{y})} \approx \frac{K_{i}}{K}
$$

Once posterior probabilities are known, classification is carried out by applying the maximum a posteriori probability rule described before. For this purpose, the $K$ nearest neighbours to the input vector are retained and the class that includes the highest number of neighbours is selected. As a result, the KNN classifier defines a non-linear decision boundary in the input space.

The smoothing parameter $K$ influences the complexity of the decision boundary defined by the classifier, with high bias (smooth boundaries) cor- 
responding to high values of $K$ (Bishop, 1995). Hence, increasing $K$ is required to ensure a reliable estimation of the posterior probabilties. On the other hand, $K$ must be sufficiently small to be sure that $p\left(\mathbf{y} \mid \omega_{i}\right)$ is similar at all the neighbours of the point $\mathbf{y}$. This trade-off leads to a compromise value of $K$ given by a small fraction of the available samples (Duda et al., 2012). According to this analysis, $K$ was set to 20 in our study, which approximately represents $1 \%$ of the images in our dataset.

\section{Results}

\subsection{Experiments on the pollen database}

In our experiments, accuracy was adopted as the performance measure to evaluate the discriminant capability of a feature pattern in the pollen classification problem. Accuracy provides the probability of correct classification for a given pollen image. This probability is approximated by the percentage of pollen grains correctly classified (Bishop, 1995). In the present study, ten-fold cross-validation was applied to estimate classification accuracy from the original dataset of 1800 images (Bishop, 1995; Duda et al., 2012).

In our experiments, several classification algorithms were implemented according to the methodology described before. Hence, in order to determine the most advantageous configuration of GLCM and LBP, different scales of analysis were evaluated for these methods. Additionally, we assessed the utility of the combination of the four texture methods in the pollen identification problem. For this purpose, among all the evaluated configurations of GLCM and LBP, those with the highest performance were selected to be combined with LGF and DTM features, resulting in the definition of a new texture 
feature pattern (ALL). Finally, to measure the impact of Fisher dimensionality reduction on the system performance, two classification schemes were compared: one using Fisher dimensionality reduction followed by the KNN algorithm (FDA-KNN), and another one using a simple KNN classifier on the raw texture features (i.e., without any kind of dimensionality reduction).

In the following Table 1, the results achieved by the classification algorithms evaluated in our experiments are summarized. Note that no result is reported for $\mathrm{LBP}_{8,1}$ with FDA-KNN classification as the dimensionality reduction stage is not applicable to feature spaces of dimension lower than $C=15$. Several observations can be made from the obtained results. First, configurations including Fisher dimensionality reduction outperformed those based on a simple KNN classifier fed with raw texture features. This reveals that the dimensionality reduction stage enabled higher generalization capability (reduced overfitting) by enforcing smooth decision boundaries between pollen categories in the input feature space. Second, the comparative analysis between the four texture methods shows that LGF and DTM yielded the highest classification performance. They achieved a correct classification rate above $92 \%$ in the pollen identification problem. $\mathrm{GLCM}_{1}$ and $\mathrm{LBP}_{\mathrm{msc}}$ were the configurations with the highest performance for GLCM and LBP methods, providing an acceptable accuracy of $89.94 \%$ and $89.83 \%$, respectively. Third, useful non-redundant texture features can be derived from GLCM and LBP analysis at different scales. Regarding the LBP method, the multiscale approach $\left(\mathrm{LBP}_{\mathrm{msc}}\right)$ outperformed single scale configurations for both FDA-KNN and KNN classification schemes. Therefore, the combination of texture features captured by LBP at different scales provides a richer de- 
scription of the texture attributes when compared to single scale analysis. In the case of GLCM, multiscale analysis led to improved classification performance when no dimensionality reduction was applied. An accuracy of $65.06 \%$ was achieved by $\mathrm{GLCM}_{\mathrm{msc}}$ while single scale configurations provided up to $60.39 \%$ accuracy. This reflects that complementary information is derived from GLCM analysis at distinct scales. However, no improvement was observed in the multiscale approach of GLCM when FDA-KNN classification was used. This result may be motivated by the increased difficulty of the dimensionality reduction task. In the multiscale approach, the dimension of the original feature space (120 features) is considerable higher than in single scale configurations (40 features), resulting in a higher degree of overlapping between categories in the transformed space (Bishop, 1995). Fourth, the combination approach (ALL) yielded $94.83 \%$ accuracy, improving the performance individually achieved by each texture method. The ALL pattern was obtained as the concatenation of $\mathrm{GLCM}_{1}, \mathrm{LGF}, \mathrm{LBP}_{\mathrm{msc}}$ and DTM patterns. Observe that, according to our results, $\mathrm{GLCM}_{1}$ and $\mathrm{LBP}_{\mathrm{msc}}$ were selected as the optimum configurations of GLCM and LBP, respectively. The result achieved by the ALL approach could be initially expected as a more robust and detailed description of the pollen texture is obtained by combining information from different methods. Specifically, we found that the ALL pattern led to 93 misclassified pollen images out of 1800 samples, what represents a high percentage of correct classification.

Table 2 details the results provided by the selected configurations of the four texture methods $\left(\mathrm{GLCM}_{1}, \mathrm{LGF}, \mathrm{LBP}_{\mathrm{msc}}\right.$ and DTM) and their combination into the ALL feature pattern. For a thorough analysis of their 


\begin{tabular}{|c|c|c|}
\hline & FDA-KNN & KNN \\
\hline $\mathbf{L B P}_{\mathbf{8 , \mathbf { 1 }}}$ & - & 81.83 \\
\hline $\mathbf{L B P}_{\mathbf{1 6 , 2}}$ & 84.89 & 80.28 \\
\hline $\mathbf{L B P}_{\mathbf{2 4 , 3}}$ & 87.83 & 80.17 \\
\hline $\mathbf{L B P}_{\text {msc }}$ & 89.83 & 84.06 \\
\hline GLCM $_{\mathbf{1}}$ & 89.94 & 57.06 \\
\hline GLCM $_{\mathbf{2}}$ & 84.39 & 57.11 \\
\hline GLCM $_{\mathbf{3}}$ & 85.83 & 60.39 \\
\hline GLCM $_{\mathbf{m s c}}$ & 85.11 & 65.06 \\
\hline $\mathbf{L G F}$ & 92.50 & 90.06 \\
\hline DTM & 92.06 & 89.83 \\
\hline ALL & 94.83 & 83.78 \\
\hline
\end{tabular}

Table 1: Total classification accuracy achieved by the evaluated texture feature patterns in the pollen identification problem. 
performance, the classification accuracy achieved on each pollen category is reported.

The results reflect that most of the errors corresponded to 'Citysus' (7) samples, for which all the methods achieved lower accuracy rates. Other categories that also presented marked difficulties in classification were 'Quercus' (13), for which LGF provided reduced accuracy, and 'Teucrium' (15), which was specially difficult for $\mathrm{GLCM}_{1}$ and $\mathrm{LBP}_{\mathrm{msc}}$. Conversely, the highest correct classification rate was achieved on 'Castanea' (5), 'Cistus' (6), 'Echium' (8) and 'Salix'(14) categories. The combination of the four texture descriptors resulted in a substantial decrease in the number of errors. For most of the categories, a positive increment in classification accuracy was observed with respect to any of the methods. It is worth noting that the increment was specially large in 'Cytisus' (7), 'Quercus' (13) and 'Aster' (1), for which an improvement higher than 5 percentage points was obtained. As observed in the individual assessment of the methods, the lowest accuracy values were still achieved on 'Cytisus' (7) and 'Teucrium' (15) samples.

\subsection{Analysis of the results}

To identify error flows between categories, Figure 4 depicts the error matrices for the four evaluated texture methods. They provide a graphical representation of the confusion matrices once the main diagonal has been removed. As a result, only the number of misclassified samples is observed. The error matrices show that 'Cytisus' (7) samples, for which all the evaluated algorithms provided reduced classification accuracy, tended to be labeled as 'Campanulaceae' (3) or 'Teucrium' (15). The similarity between 'Cytisus' (7) and 'Teucrium' (15) pollen samples also led to a significant number of 


\begin{tabular}{|c|c|c|c|c|c|c|}
\hline & \multicolumn{5}{|c|}{ Texture Features } \\
\hline & & $\mathrm{GLCM}_{1}$ & LGF & $\mathrm{LBP}_{\mathrm{msc}}$ & DTM & ALL \\
\hline \multirow{16}{*}{ 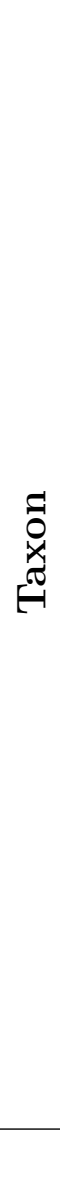 } & Aster & 89.17 & 95.00 & 94.17 & 91.67 & 100.00 \\
\hline & Brassica & 95.83 & 89.17 & 82.50 & 94.17 & 90.00 \\
\hline & Campanulaceae & 85.83 & 93.33 & 86.67 & 92.50 & 90.83 \\
\hline & Carduus & 98.33 & 97.50 & 89.17 & 95.83 & 99.17 \\
\hline & Castanea & 99.17 & 100.00 & 100.00 & 99.17 & 100.00 \\
\hline & Cistus & 99.17 & 99.17 & 97.50 & 99.17 & 99.17 \\
\hline & Cytisus & 73.33 & 77.50 & 69.17 & 71.67 & 85.83 \\
\hline & Echium & 94.17 & 98.33 & 98.33 & 99.17 & 98.33 \\
\hline & Ericaceae & 95.00 & 89.17 & 93.33 & 88.33 & 99.17 \\
\hline & Helianthus & 81.67 & 93.33 & 90.83 & 94.17 & 90.83 \\
\hline & Olea & 93.33 & 91.67 & 91.67 & 92.50 & 96.67 \\
\hline & Prunus & 98.33 & 99.17 & 92.50 & 93.33 & 96.67 \\
\hline & Quercus & 88.33 & 81.67 & 90.83 & 87.50 & 95.00 \\
\hline & Salix & 95.00 & 95.83 & 95.83 & 96.67 & 99.17 \\
\hline & Teucrium & 62.50 & 85.83 & 75.00 & 85.00 & 81.67 \\
\hline & Total & 89.94 & 92.50 & 89.83 & 92.06 & 94.83 \\
\hline
\end{tabular}

Table 2: Accuracy on each pollen category and total accuracy for the most relevant classification algorithms. 
classification errors in the opposite direction. Hence, a common trend was observed in the four evaluated methods since misclassified 'Teucrium' (15) images were usually labelled as 'Cytisus' (7) by all of them. In addition to the common difficulties found with 'Cytisus' (7) samples, other particular results were observed. For instance, LGF and LBP labelled as 'Olea' (11) a relevant number of 'Quercus' (13) and 'Brassica' (2) samples, respectively. In addition, GLCM and LBP exhibited particular difficulties between 'Aster' (1) and 'Healianthus' (10).

The detected error flows reflect a coherent behaviour of our classification algorithms. As shown in Figure 1, these flows connect pollen taxa with a similar appearance. As an example, consider the pairs given by 'Cytisus' (7) and 'Teucrium' (15), 'Aster' (1) and 'Healianthus' (10), or 'Brassica' (2) and 'Olea' (11). At this point, it is interesting to analyze the effect of the combination approach on the observed error flows. Table 3 details the confusion matrix obtained when a single feature pattern with all the texture features is used to classify pollen samples. As can be appreciated, most of the errors associated with each individual texture method are removed by adopting the combination approach. Indeed, only the error flow between 'Cytisus' (7) and 'Teucrium' (15) seems to be substantial. Therefore, the combination of different texture features provided the most efficient characterization of the pollen taxon.

According to our results, LGF and DTM capture more useful information about the pollen taxon, resulting in higher classification accuracy than the other texture descriptors. These two methods are based on the analysis of the image using a bank of filters placed at different frequency bands (Field 


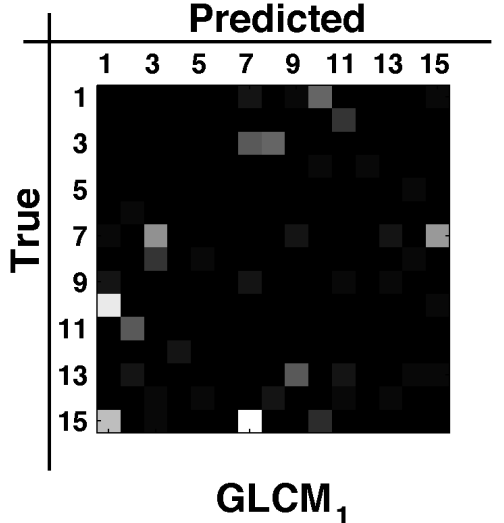

(a)

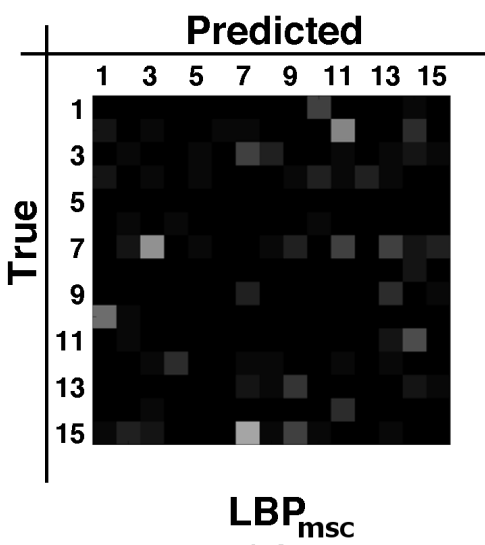

(c)

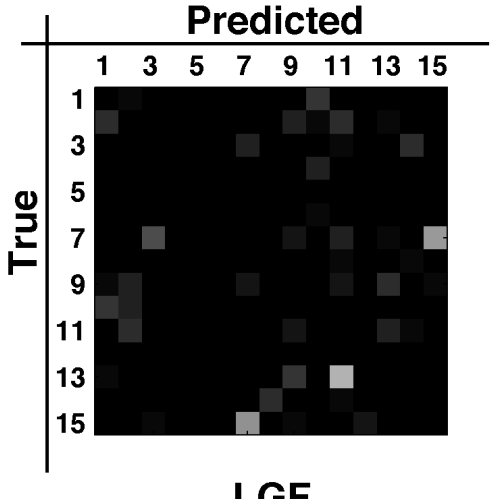

(b)

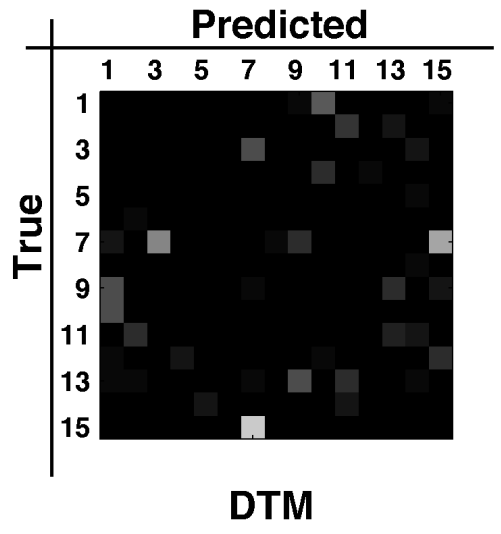

(d)

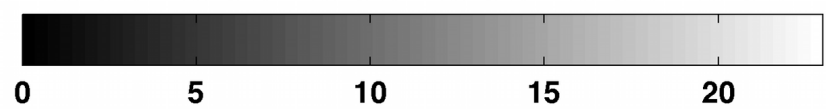

Figure 4: Error matrices for each texture method. 


\begin{tabular}{|c|c|c|c|c|c|c|c|c|c|c|c|c|c|c|c|}
\hline \multicolumn{1}{c|}{} & \multicolumn{10}{c|}{ Predicted } \\
\hline & $\mathbf{1}$ & $\mathbf{2}$ & $\mathbf{3}$ & $\mathbf{4}$ & $\mathbf{5}$ & $\mathbf{6}$ & $\mathbf{7}$ & $\mathbf{8}$ & $\mathbf{9}$ & $\mathbf{1 0}$ & $\mathbf{1 1}$ & $\mathbf{1 2}$ & $\mathbf{1 3}$ & $\mathbf{1 4}$ & $\mathbf{1 5}$ \\
\hline $\mathbf{1}$ & 120 & & & & & & & & & & & & & & \\
\hline $\mathbf{2}$ & & 108 & & & & & & & & & 12 & & & & \\
\hline $\mathbf{3}$ & & & 109 & & & & 8 & & & & & & & 3 & \\
\hline $\mathbf{4}$ & & & & 119 & & & & & & & & 1 & & & \\
\hline $\mathbf{5}$ & & & & & 120 & & & & & & & & & & \\
\hline $\mathbf{6}$ & & & & & & 119 & & & & & 1 & & & & \\
\hline $\mathbf{7}$ & & & 7 & & & & 103 & & 1 & & & & & 1 & 8 \\
\hline $\mathbf{8}$ & & & & & & & & 118 & & & & & & 2 & \\
\hline $\mathbf{9}$ & & & & & & & 1 & & 119 & & & & & & \\
\hline $\mathbf{1 0}$ & 10 & & & & & & & & & 109 & & 1 & & & \\
\hline $\mathbf{1 1}$ & & 3 & & & & & & & & & 116 & & & 1 & \\
\hline $\mathbf{1 2}$ & 1 & & & 1 & & & & & & 2 & & 116 & & & \\
\hline $\mathbf{1 3}$ & & 1 & & & & & 1 & & 2 & & & & 114 & 2 & \\
\hline $\mathbf{1 4}$ & & & & & 1 & & & & & & & & & 119 & \\
\hline $\mathbf{1 5}$ & & & 1 & & & & 20 & & 1 & & & & & & 98 \\
\hline
\end{tabular}

Table 3: Confusion matrix resulted from the combination of the four texture methods. 
et al., 1987; Marcos and Cristóbal, 2013). This suggests that the spectral content of a pollen image will vary from a category to another while it will be similar for samples in the same category. To analyze this behaviour, we computed the average power spectral density (PSD) of pollen images in each category, which is depicted in Figure 5. The spectra exhibit approximate radial symmetry, indicating that the pollen textures are not associated with a specific orientation. In addition, the resulting figure shows that the energy of a pollen image is composed of different frequency components depending on its taxon. For instance, high frequency components are observed in 'Cistus' (6) pollen images while 'Aster' (1) and 'Campanulaceae' (3) images do not have a siginificant energy content at high frequencies. On the other hand, 'Castanea' (5) images tend to have lower energy than pollen samples from other taxa as reflected by the small magnitude of the averaged spectrum. To appreciate these differences, Figure 5 shows the profile of the PSD along the $x$-axis (i.e., the frequency coordinate $f_{y}$ is set to 0 ). It is worth noting that the choice of the orientation does not have a significant influence on this analysis as the spectra do not reveal substantial variations along the angle coordinate. Categories 'Campanulaceae' (3), 'Cytisus' (7) and 'Teucrium' (15), among which the evaluated methods performed most of the classification errors, have very similar PSD profiles. On the contrary, other categories such as 'Castanea' (5), 'Cistus' (6) or 'Echium' (8) exhibit a profile significantly different to the rest. As a result, they can be more easily identified as reflected by the classification accuracy close to $100 \%$ achieved by LGF and DTM on these categories. 

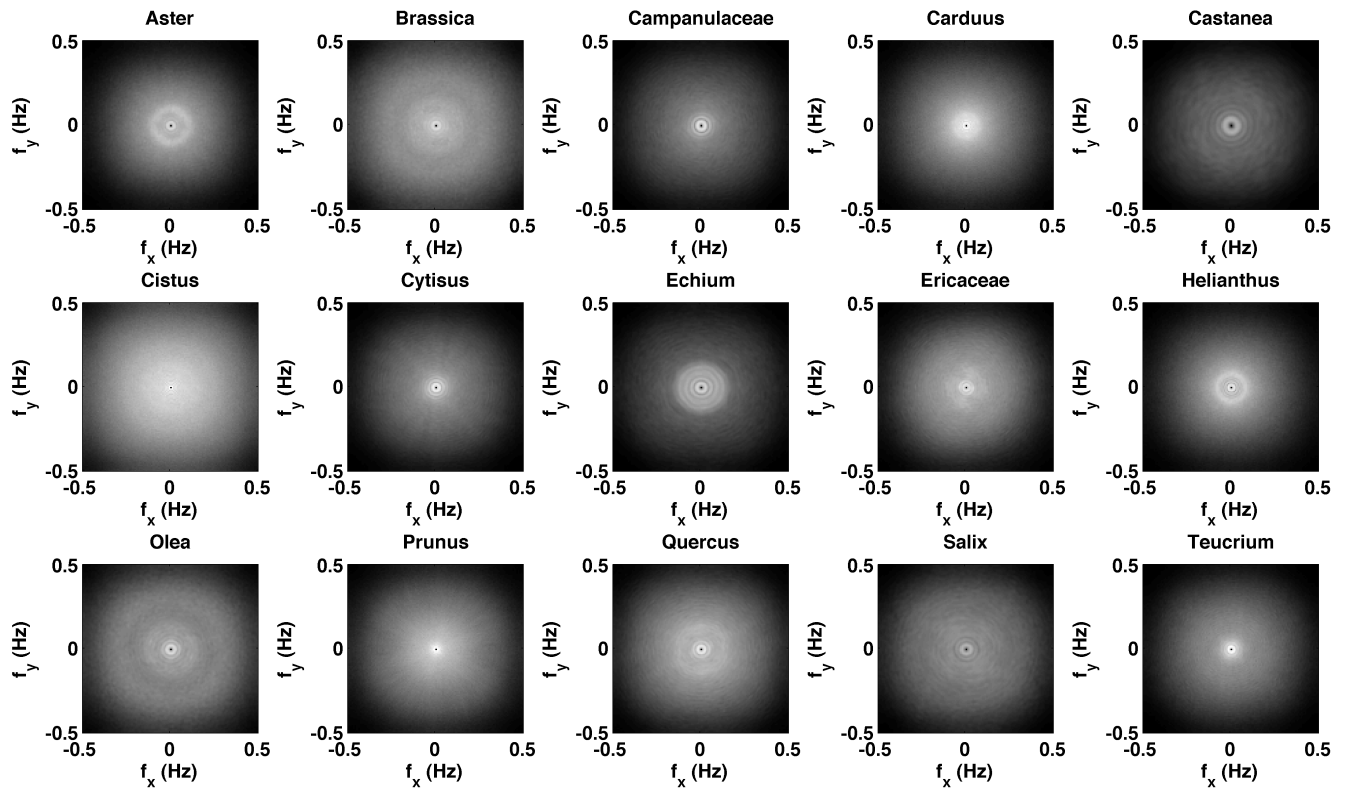

(a)
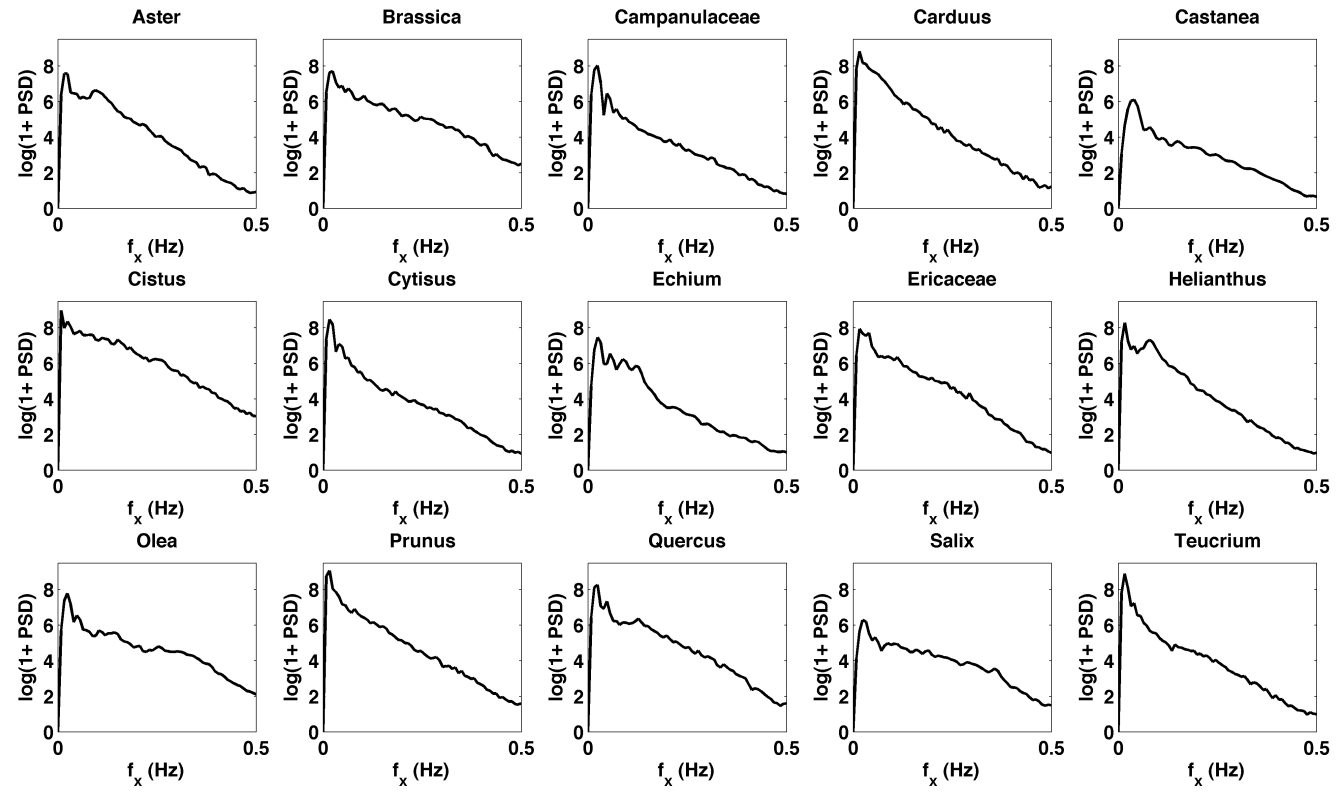

(b)

Figure 5: Spectral content of pollen images in each category: (a) averaged PSD of images in each category and (b) profile of the averaged PSD along the $f_{x}$ coordinate. 


\section{Discussion and conclusions}

A detailed analysis on the role of texture in pollen taxon characterization was presented. In addition to conventional texture feature extraction based on GLCM, other methods including LGF, LBP and DTM were assessed in our study. Furthermore, the complementarity between these texture features was explored by means of their combination. A methodology involving dimensionality reduction based on FDA and KNN classification was proposed to identify pollen samples from 15 different taxa. The obtained results reflect the superior performance of LGF and DTM texture descriptors as well as the utility of the combination approach in the proposed pollen classification problem.

Our experiments support the conclusion that texture is a distinctive characteristic of the pollen taxon. Note that $95 \%$ of the grains in a dataset composed of 1800 samples from 15 different taxa were correctly identified by only using texture features. In particular, it should be appreciated that texture analysis enabled high discrimination between pollen samples from taxa with an increased degree of similarity. For instance, consider the pairs given by 'Brassica' (2) and 'Olea' (11) as well as 'Aster' (1) and 'Helianthus' (10) (see Figure 1). In the case of 'Cytisus' (7) and 'Teucrium' (15), which were responsible for most of the errors of our algorithms, a significant classification accuracy over $80 \%$ has been achieved. On the other hand, we have demonstrated that pollen textures from distinct categories tend to exhibit differences in their spectral content. Hence, texture descriptors based on spectral analysis such as LGF and DTM have shown to be capable of capturing these differences, reaching significantly high accuracy in the pollen clas- 
sification problem. However, we found that the most effective representation of the pollen taxa was not given by a single texture descriptor. Instead, the combination of different texture features resulted in improved classification accuracy. This represents a more complex approach since several processing techniques must be applied to perform texture feature extraction from the original pollen image. Nevertheless, our results show that uncorrelated texture features complement themselves in order to enable the pollen identification task.

Previously, other researchers highlighted the relevance of texture in pollen identification. Commonly, GLCM analysis was proposed to extract texture attributes (Langford et al., 1990; Li and Flenley, 1999; Kaya et al., 2013; Rodriguez-Damian et al., 2006; Zhang et al., 2004, 2005). These features were applied individually or in combination with other descriptors for automatic pollen classification. The achieved accuracies ranged from 88\% (10 categories) (Kaya et al., 2013) to 100\% (4 categories) (Li and Flenley, 1999). A fair comparison of our results with those reported in preceding studies cannot be carried out since different databases were analyzed. However, two main observations can be made. First, a comparable or higher number of samples and categories was included in our research. This contributes to increase the reliability of our experimental results and reflects the validity of the proposed texture methods in the analysis of microscopy pollen images. Second, although most of the previous studies used GLCM for texture characterization, other texture descriptors such as LGF or DTM based on the spectral properties of the pollen image have proven to be more efficient for capturing distinctive information about the taxa. 
The main handicap for the development of an automatic pollen identification method is the huge number of distinct plant species and pollen taxa. As a result, the implementation of a universal method for pollen recognition seems an intractable task. Instead, a subset of pollen taxa including those involved in a specific application or context is usually considered. In our study, 15 different taxa correspoding to honey-bee pollen from the mediterranean area were analyzed. However, it must be noted that the evaluated texture methods can be applied to other domains in which automatic pollen identification is required. Indeed, texture features provide several advantages when compared with other techniques for this purpose. For instance, unlike shape or morphological features, texture analysis adapts to the evaluation of fossil pollen samples that may be broken (Li et al., 2004). In addition, texture avoids the dependence on the position of the pollen grain, which is crucial for methods based on the detection of pores. Furthermore, no complex equipment is required to acquire pollen images such as those evaluated in our study, which were captured through brightfield microscopy. Hence, the proposed methods could be implemented in a specific software module coupled to the microscope, enabling the analysis of a large number of pollen samples in a reasonable time. This would overcome the limitation of spectroscopy techniques (Pappas et al., 2003; Ivleva et al., 2005; DellAnna et al., 2009; Schulte et al., 2008), which require more complex optical elements (spectrometers) and preparation to analyze the composition of the pollen samples.

Despite the advantages from texture analysis, several limitations can be found in our study. Although the available image dataset includes a relevant 
number of taxa and samples, its size seems to be insufficient for certain tasks involving the implementation of the classification algorithm. For instance, we adopted KNN with $K=20$ for multivariate pattern classification. A search for the optimum $K$ was not carried out as an independent dataset would be needed to avoid biased test results. In addition, previous studies in the field reported significant peformance by means of neural networks or support vector machines ( $\mathrm{Li}$ and Flenley, 1999; Rodriguez-Damian et al., 2006). Nevertheless, these algorithms have a considerable amount of design parameters and adaptable weights. A larger number of samples per category than that available in our research would be required to adjust them and prevent overfitting (Bishop, 1995). The latter must be taken into account in the design of classification algorithms. In our study, the strategy adopted to avoid overfitting was the use of dimensionality reduction based on FDA. Our experiments revealed that dimensionality reduction played an essential role to obtain classification algorithms with a high generalization capability. As an alternative, other procedures could have been considered in order to reduce the number of input features to the classifier. For instance, a smaller number of statistics could be derived from each of the filters employed in LGF or GLCM features could be averaged along the four angle directions. Another limitation of our research is given by the acquisition of the data, as images of isolated pollen grains analyzed in our study were manually obtained from the image of the whole slide. Further research is required in order to achieve a fully automatic system for pollen identification by implementing a software module enabling the localization of pollen grains in the original slide. Finally, it is worth noting that a single image was analyzed from the 
whole stack captured for each pollen grain. Future studies could evaluate the use of fusion techniques including several images at different distances from the object.

In summary, our study reflects that the texture of the pollen exine is a distinguishing property of its taxon. According to our results, other methods are more useful for the characterization of the pollen texture than conventional first-order and secon-order statistics such as Haralick's GLCM. Specifically, we have shown that the spectral representation of pollen images enables the extraction of texture features that vary from a taxon to another. Thus, texture descriptors based on spectral filtering such as LGF or DTM suitable adapt to the pollen identification problem. Furthermore, our experiments reveal that the complementarity between different texture features can be exploited in order to achieve higher classification performance. Therefore, we propose an exhaustive analysis of texture in image-based applications pursuing automatic identification of pollen taxa.

\section{Acknowledgments}

This work has been partially supported by the EU-funded "Apifresh" Project coordinated by "Inspiralia" (http://www.apifresh.eu). J. V. Marcos is a research fellow at Institute of Optics (CSIC) under the programme Juan de la Cierva (Spanish Ministry of Economy and Competitiveness). R. Nava thanks Consejo Nacional de Ciencia y Tecnologa (CONACYT) and PAPIIT grant IG100814. 


\section{References}

Bishop, C.M., 1995. Neural networks for pattern recognition. Oxford University Press, Oxford.

Boucher, A., Hidalgo, P.J., Thonnat, M., Belmonte, J., Galan, C., Bonton, P., Tomczak, R., 2002. Development of a semi-automatic system for pollen recognition. Aerobiologia 18, 195-201.

Carrión, P., Cernadas, E., Gálvez, J.F., Damián, M., de Sá-Otero, P., 2004. Classification of honeybee pollen using a multiscale texture filtering scheme. Mach. Vis. Appl. 15, 186-193.

Chen, C., Hendriks, E.A., Duin, R.P., Reiber, J.H., Hiemstra, P.S., de Weger, L.A., Stoel, B.C., 2006. Feasibility study on automated recognition of allergenic pollen: grass, birch and mugwort. Aerobiologia 22, 275-284.

Chica, M., 2012. Authentication of bee pollen grains in bright-field microscopy by combining one-class classification techniques and image processing. Microsc. Res. Tech. 75, 1475-1485.

Das, D.K., Ghosh, M., Pal, M., Maiti, A.K., Chakraborty, C., 2013. Machine learning approach for automated screening of malaria parasite using light microscopic images. Micron 45, 97-106.

DellAnna, R., Lazzeri, P., Frisanco, M., Monti, F., Campeggi, F.M., Gottardini, E., Bersani, M., 2009. Pollen discrimination and classification by Fourier transform infrared (FT-IR) microspectroscopy and machine learning. Anal. Bioanal. Chem. 394, 1443-1452. 
Duda, R.O., Hart, P.E., Stork, D.G., 2012. Pattern classification. John Wiley \& Sons, New York.

Erdtman, G., Sarjeant, W.A.S., Praglowski, J., Nilsson, S., Dunbar, A., 1969. Handbook of palynology: morphology, taxonomy, ecology. An introduction to the study of pollen grains and spores. Hafner, New York.

Field, D.J., et al., 1987. Relations between the statistics of natural images and the response properties of cortical cells. J. Opt. Soc. Am. A 4, 2379 2394.

Fisher, R.A., 1936. The use of multiple measurements in taxonomic problems. Annals of eugenics 7, 179-188.

Gao, X., Sattar, F., Venkateswarlu, R., 2007. Multiscale corner detection of gray level images based on log-Gabor wavelet transform. IEEE Trans. Circuits Syst. Video Technol. 17, 868-875.

Gross, M.H., Koch, R., 1995. Visualization of multidimensional shape and texture features in laser range data using complex-valued Gabor wavelets. IEEE Trans. Visual. and Comput. Graphics 1, 44-59.

Haralick, R.M., Shanmugam, K., Dinstein, I.H., 1973. Textural features for image classification. IEEE Trans. Syst. Man Cybern. , 610-621.

Ivleva, N., Niessner, R., Panne, U., 2005. Characterization and discrimination of pollen by Raman microscopy. Anal. Bioanal. Chem. 381, 261-267.

Kaya, Y., Erez, M.E., Karabacak, O., Kayci, L., Fidan, M., 2013. An automatic identification method for the comparison of plant and honey pollen 
based on GLCM texture features and artificial neural network. Grana 52, $71-77$.

Kovesi, P., 1999. Image features from phase congruency. VIDERE: Journal of computer vision research 1, 1-26.

Kumar, S., Ong, S.H., Ranganath, S., Chew, F.T., 2010. Invariant texture classification for biomedical cell specimens via non-linear polar map filtering. Comput. Vis. Image Underst. 114, 44-53.

Langford, M., Taylor, G., Flenley, J., 1990. Computerized identification of pollen grains by texture analysis. Rev. Palaeobot. Palynology 64, 197-203.

Li, P., Flenley, J.R., 1999. Pollen texture identification using neural networks. Grana 38, 59-64.

Li, P., Treloar, W., Flenley, J., Empson, L., 2004. Towards automation of palynology 2: the use of texture measures and neural network analysis for automated identification of optical images of pollen grains. J. Quat. Sci. 19, $755-762$.

Marcos, J.V., Cristóbal, G., 2013. Texture classification using discrete Tchebichef moments. J. Opt. Soc. Am. A 30, 1580-1591.

Mitsumoto, K., Yabusaki, K., Aoyagi, H., 2009. Classification of pollen species using autofluorescence image analysis. J. Biosci. Bioeng. 107, 9094.

Mukundan, R., 2004. Some computational aspects of discrete orthonormal moments. IEEE Trans. Image Process. 13, 1055-1059. 
Mukundan, R., Ong, S., Lee, P.A., 2001. Image analysis by Tchebichef moments. IEEE Trans. Image Process. 10, 1357-1364.

Nava, R., Escalante-Ramírez, B., Cristóbal, G., 2012. Texture image retrieval based on log-Gabor features, in: Progress in Pattern Recognition, Image Analysis, Computer Vision, and Applications. Springer, Berlin, pp. 414421.

Ojala, T., Pietikainen, M., Maenpaa, T., 2002. Multiresolution gray-scale and rotation invariant texture classification with local binary patterns. IEEE Trans. Pattern Anal. Mach. Intell. 24, 971-987.

Otsu, N., 1975. A threshold selection method from gray-level histograms. Automatica 11, 23-27.

Pappas, C., Tarantilis, P., Harizanis, P., Polissiou, M., 2003. New method for pollen identification by FT-IR spectroscopy. Appl. Spectrosc. 57, 23-27.

Punyasena, S.W., Tcheng, D.K., Wesseln, C., Mueller, P.G., 2012. Classifying black and white spruce pollen using layered machine learning. New Phytol. 196, 937-944.

Randen, T., Husoy, J.H., 1999. Filtering for texture classification: a comparative study. IEEE Trans. Pattern Anal. Mach. Intell. 21, 291-310.

Rodriguez-Damian, M., Cernadas, E., Formella, A., Fernández-Delgado, M., De Sa-Otero, P., 2006. Automatic detection and classification of grains of pollen based on shape and texture. IEEE Trans. Syst. Man Cybern. Part C-Appl. Rev. 36, 531-542. 
Schulte, F., Lingott, J., Panne, U., Kneipp, J., 2008. Chemical characterization and classification of pollen. Anal. Chem. 80, 9551-9556.

Schwartz, W.R., de Siqueira, F.R., Pedrini, H., 2012. Evaluation of feature descriptors for texture classification. J. Electron. Imaging 21, 023016-1.

Soh, L.K., Tsatsoulis, C., 1999. Texture analysis of SAR sea ice imagery using gray level co-occurrence matrices. IEEE Trans. Geosci. Remote Sensing $37,780-795$.

Stillman, E., Flenley, J.R., 1996. The needs and prospects for automation in palynology. Quat. Sci. Rev. 15, 1-5.

Teh, C.H., Chin, R.T., 1988. On image analysis by the methods of moments. IEEE Trans. Pattern Anal. Mach. Intell. 10, 496-513.

Treloar, W., Taylor, G., Flenley, J., 2004. Towards automation of palynology 1: analysis of pollen shape and ornamentation using simple geometric measures, derived from scanning electron microscope images. J. Quat. Sci. $19,745-754$.

Tuceryan, M., Jain, A.K., 1993. Texture analysis, in: Chen, C., Pau, L., Wang, P. (Eds.), The handbook of pattern recognition and computer vision. World Scientific, Singapore.

Zhang, Y., Fountain, D., Hodgson, R., Flenley, J., Gunetileke, S., 2004. Towards automation of palynology 3: pollen pattern recognition using Gabor transforms and digital moments. J. Quat. Sci. 19, 763-768. 
Zhang, Y., Wang, R., Hunter, P., 2005. Airborne pollen texture discrimination using wavelet transforms in combination with cooccurrence matrices. International Journal of Intelligent Systems Technologies and Applications 1, 143-156.

\section{Figure captions}

Figure 1. Sample images of the pollen taxa analyzed in our study.

Figure 2. A scheme of the methodology adopted for automatic identification of the pollen taxon based on texture analysis. From the input image, four main processing stages are identified: segmentation and preprocessing, texture feature extraction, dimensionality reduction and classification.

Figure 3. Segmentation proccess for the identification of the pollen grain in the input image.

Figure 4. Error matrices for the four evluated texture methods.

Figure 5. Spectral content of pollen images in each category: (a) averaged PSD of images in each category and (b) profile of the averaged PSD along the $f_{x}$ coordinate.

\section{Table captions}

Table 1. Total classification accuracy achieved by the evaluated texture feature patterns in the pollen identification problem.

Table 2. Accuracy on each pollen category and total accuracy for the most relevant classification algorithms.

Table 3. Confusion matrix resulted from the combination of the four texture methods. 